\title{
LA FORMACIÓN DEL FEUDALISMO EN VALDEORRAS: EL NACIMIENTO DE LAS PRIMERAS ESTRUCTURAS FEUDALES: SIGLOS X - MEDIADOS DEL XII
}

\author{
por \\ MARÍA DEL CARMEN GÓMEZ BAJO
}

\section{PRELIMINARES}

De entrada ha de reconocerse que el tema elegido presenta sumas dificultades a la vez que encierra una gran complejidad. Las razones son diversas. Tenemos una zona: Valdeorras, un momento crolonógico muy determinado: siglos X - mediados del XII, y un tema muy específico: la formación de las primeras estructuras feudales en la citada zona.

Si partimos primeramente del tema, es decir de la formación del Feudalismo, es de sobra conocido que hasta hace no mucho tiempo ha permanecido en el olvido. No era el foco de mira y de atención por parte de los investigadores, pues en tanto se partía del principio de su negativa en España a excepción del área catalana, obviamente no se iba a investigar sobre algo que "a priori" no existía!.

Esto en principio ya supone un obstáculo, si bien hay que matizar que cada día son ya muchos los estudios que tratan de demostrar la existencia

\footnotetext{
' Teoría mantenida a lo largo del tiempo por la corriente institucionalista a cuya cabeza se encontraba Sánchez Albornoz. Vid. obra del citado autor "Pequeños propietarios libres en el reino astur-leonés", Spoleto, 1965.
}

"CUADERNOS DE ESTUDIOS GALLEGOS", Tomo XLI, Fascículo 106, Santiago 1993-94. 
del feudalismo no sólo en Cataluña, sino en el resto de España ${ }^{2}$; sin embargo aún queda por hacer en muchos campos dentro de la especificidad propia del tema.

Es una limitación que se debe de tener en cuenta. No obstante con este análisis tenemos la esperanza de poder llegar a algunas conclusiones lo más fiables posibles. Se ha de adelantar que en ocasiones habrá que recurrir a hipótesis sustentadas en una considerable labor hermeneútica. Somos conscientes de que son susceptibles de una crítica más severa, pero en último término estimamos que no sólo no perjudican a la investigación, sino que por más novedosas contribuyen a su avance y logran una historia cada vez más epistemológica.

Otra limitación, tal vez más severa la impone la propia zona. Normalmente todo investigador del Medievo se encuentra con una dificultad que a veces es insalvable y es la limitación de las Fuentes. No es muy usual que se conserve abundante documentación en general para toda la época Medieval, y aún menos para el momento altomedieval.

La zona que pretendemos estudiar presenta serias dificultades en este aspecto. En primer lugar hay que advertir que se cuenta con muy poca documentación. No es Valdeorras una zona al estilo de otras vecinas como puede ser el Bierzo, que ofrezca posibilidades proverbiales para su estudio. Las fuentes, muy escasas, no lo facilitan, pero tampoco la especificidad propia de la zona.

Mientras que para el Bierzo se constatan el surgimiento de un número considerable de Monasterios en la fase Altomedieval $^{3}$, así como de una efectiva labor llevada a cabo por monarcas y magnates junto a comunida-

\footnotetext{
${ }^{2}$ La tendencia más general es la de demostrar a través de estudios que se centran en núcleos locales la existencia de Feudalismo. Por citar algunos ejemplos entre un sin fin de obras, es de destacar el análisis que para Tierra de Campos Occidental ha realizado P. Martínez Sopena "La tierra de Campos Occidental: Poblamiento, Poder y Comunidads. X-XIII", Valladolid, 1985.

En la misma línea, pero en este caso para la zona de Ávila, el estudio de A. Barrios García "Estructuras Agrarias y de poder en Castilla: el ejemplo de Ávila (1085-1320)", Salamanca 1983, T. II.

La ciudad de León cuenta con un magnífico estudio a cargo del profesor Estepa "Estructura social de la ciudad de León (siglos X-XIII)", León 1977.

${ }^{3}$ Remitimos al lector a nuestra Tesis Doctoral "El Bierzo en los siglos XII-XIV: Señores y Campesinos", Facultad de Filosofía y Letras León, Departamento de Historia Medieval. Actualmente se está ultimando su revisión para su próxima publicación.
}

"CUADERNOS DE ESTUDIOS GALLEGOS", Tomo XLI, Fascículo 106, Santiago 1993-94. 
des campesinas en la primera etapa de "colonización"4, para Valdeorras en este sentido conocemos muy poco.

No es precisamente Valdeorras una Tebaida Berciana. El número de cenobios en la misma quedó reducido exclusivamente a tres en el período medieval "San Martín de Alixo junto con el de San Salvador o San Miguel de Cobas y Santa Cruz de Casoio", nos dice un estudio realizado sobre la zona que fueron de los pocos monasterios constatados en Valdeorras ${ }^{5}$. Hablamos en el sentido de Monasterios ubicados como tal en la zona.

Ahora bien, se conoce la importancia de la zona como foco de atención por parte de poderes feudales que próximos a ella — concretamente en el Bierzo-, deciden ubicar propiedades en la misma. En tal sentido podemos hablar del Monasterio de San Pedro de Montes situado en la zona Sur de la comarca berciana, concretamente en la parte de los Montes Aquilanos. Tal vez sea uno de los poderes señoriales que indirectamente ejerció y desempeñó un importante papel en la zona objeto de estudio ${ }^{6}$.

En el mismo sentido, si bien con menor importancia, podemos hablar del Monasterio de Santa María de Carracedo, en pleno corazón de la hoya berciana. Algunos documentos conservados para el mismo nos remiten a la zona de Valdeorras ${ }^{7}$. Otros como el Monasterio de San Andrés de Vega

\footnotetext{
${ }^{4}$ Algunas donaciones nos hablan por ejemplo de la importancia del monarca en este momento. Así en 992. Vermudo II dona al Monasterio de Santa Leocadia de Castañeda siervos y heredades en distintas villas del Bierzo. A.H.N.s. Clero, Códices, Códice 970, fol. 14.

Otras donaciones protagonizadas por nobles nos remiten a esta misma importancia. Así en 983 Ecta Avivez y su mujer Rachel donan al Monasterio de S. Cosme y S. Damián la villa "Pandello y su iglesia de San Clemente". B.N.Mss 4357, Tbo Negro, n 358. En esta misma línea en el año 993 Jimeno Velaz efectúa una amplia donación al Monasterio de Santa Leocadia de Castañeda. Entre los bienes donados se encuentran varias villas fuera y dentro del Bierzo, concretamente se explicita "la villa de Toreno", Ibid, n. 418. Muchos más documentos nos hablan en este sentido. Remitirse a nuestra Tesis Doctoral citada, Cap. III.

${ }^{5}$ García Tato I., Dominios del Monasterio de San Pedro de Montes en Valdeorras, Cadernos Instituto de Estudios Vadeorrenses, n. 7, p. 58.

${ }^{6}$ Nos apoyaremos para seguir su estudio en el tumbo que ha publicado Quintana Prieto A. "Tumbo Viejo de San Pedro de Montes", León 1971.

${ }^{7}$ El tumbo del Monasterio de Santa María de Carracedo se encuentra en el A.D.A. Ha sido hecho por una copista del siglo XVIII y consta de un índice de escrituras, bulas, donaciones, etc... cita el tumbo "que se encuentra este cartulario según la enumeración de Cajones del Archivo del Monasterio de Carracedo". Consta de 549 documentos.
}

"CUADERNOS DE ESTUDIOS GALlEGOS", Tomo XLI, Fascículo 106, Santiago 1993-94. 
de Espinareda, en la parte septentrional berciana ${ }^{8}$, o el de Samos, ya en Galicia $^{9}$, nos hablan de una escasa influencia en Valdeorras si bien no la abandonan.

Un Monasterio como puede ser el de San Martín de Castañeda, en Sanabria ${ }^{10}$, totalmente alejado de Valdeorras, desempeñó un notable papel en Valdeorras muy en la línea del ejercido por el Monasterio de Santa María de Carracedo. Todos ellos serán los que indirectamente nos permitirán seguir en la medida de la posible y a través de su influencia la formación de las estructuras feudales en Valdeorras. Sería mucho más interesante contar en la zona con poderes señoriales directamente allí asentados, pues ello nos situaría ante realidades más evidentes amén de que nos facilitaría en cierto modo la labor a realizar.

Cabe a continuación exponer el objetivo concreto que perseguimos con este estudio, si bien subrepticiamente ya lo hemos dejado entrever.

Como hemos adelantado pretendemos llegar a conocer el nacimiento del Feudalismo en Valdeorras. Así pues dividiremos el estudio cronológicamente en dos períodos. Un primer período - período asturenglobará todo el siglo $\mathrm{X}$. El segundo período partirá de fines del siglo $\mathrm{X}$ y se extenderá hasta mediados del siglo XII. Será ésta una etapa de camino hacia la consolidación del Feudalismo. Consolidación que nosotros situamos cronológicamente a partir de mediados del siglo XII y hasta principios del siglo XV. No entra dentro de nuestro cometido el análisis de dicha consolidación, pero sí manifestamos nuestro deseo de llevarlo a cabo en otro momento.

Por otro lado y en base a que hemos dedicado nuestra Tesis Doctoral al estudio del Bierzo en la Edad Media" ${ }^{11}$, proponemos como trabajo a reali-

\footnotetext{
${ }^{8}$ Algunos documentos del Tumbo citado de San Pedro de Montes nos hablan de esta relativa importancia. Vid. Dcto. n. 311 y 362 . Por lo que respecta a la Documentación del Monasterio de San Andrés de Vega de Espinareda, de 186 Documentos transcritos para los siglos XII-XIV, ninguno nos hace referencia a Valdeorras. Gómez Bajo, C. Documentación Medieval del Monasterio de S. Andrés de Vega de Espinareda (León) (siglos XII$X I V)$. Edic. Universidad de Salamanca y León. Salamanca 1993.

${ }^{9}$ Son mínimas las referencias a Valdeorras en el Tumbo de San Julián de Sampos (siglos VIII-XII) de M. Lucas Alvarez, Santiago de Compostela, 1986, vid. dcto. S. 2.

${ }^{10}$ García Tato I., La Documentación medieval del Tumbo del Monasterio de S. Martín de Castañeda relativa a Valdeorras. O Barco de Valdeorras, 1986.

"Gómez Bajo M.C.: El Bierzo en los siglos XII-XIV: Señores y Campesinos. Dirigida por el Dr. C. Estepa Díez y presentada en la F.F y Letras de León en Junio de 1988.
} 
zar dentro de este objetivo el cotejo de ambas zonas siempre que las circunstancias así lo permitan, pero sin viciarnos en este sentido, pues entendemos que la base fundamental de análisis se centra en Valdeorras.

\section{CONCEPTUALIZACIÓN SOBRE LA FORMACIÓN DEL FEU- DALISMO}

Para comprender en su integridad la formación del Feudalismo, hemos de tratar dos aspectos de sumo interés. Uno de ellos en cierta manera ya lo hemos adelantado y se refiere a esa negativa que a lo largo del tiempo se ha mantenido en torno a la no existencia del Feudalismo en la Península Ibérica a excepción de Cataluña.

El mismo indirectamente nos obliga a centrarnos en otro tema como derivación de éste, en tanto uno y otro están estrechamente relacionados y en última instancia son consecuencia uno del otro. Nos estamos refiriendo a lo que se ha denominado fase de colonización o más concretamente de repoblación, necesaria fundamentalmente tras las campañas de Alfonso I (739-757) en el valle del Duero.

La corriente institucionalista a cuya cabeza se encontraba Sánchez Albornoz ha mantenido durante mucho tiempo la teoría de que con objeto de las campañas llevadas a cabo por Alfonso I, los territorios de la Meseta Norte fueron vaciados de población fruto de una auténtica conquista contra los musulmanes, a los que exterminó dicho monarca, y se retiró con los efectivos cristianos allí asentados a su núcleo de origen: el reino astur. Todo ello produciría un auténtico vacío de población en el valle del Duero y por consiguiente todas las incursiones o toma de ciudades protagonizadas inicialmente por Ordoño I y continuadas por sus sucesores en la expansión del reino astur hacia el Duero, implicarían una ocupación y entrada en el vacío en zonas totalmente deshabitadas. Una obra exclusivamente ha dedicado S. Albornoz a este tema ${ }^{12}$ y que en principio a lo largo del tiempo ha tenido muchos seguidores.

Sin embargo hoy cada vez es menos admitida esta teoría, y son cada vez más los investigadores encargados de refutarla. Pionero de los mismos en contra de una auténtica despoblación ha sido Menéndez Pidal.

${ }^{12}$ C. Sánchez Albornoz: Despoblación y Repoblación del Valle del Duero, Buenos Aires, 1965.

"CUADERNOS DE ESTUdios GALLEGOS", Tomo XLI, Fascículo 106, Santiago 1993-94. 
Para él poblar no significaría pasar a ocupar algo deshabitado, entrar en el vacío, sino dominar. Se trataría de que algo que carecía de organización, a partir de ese momento pasaba a tenerla ${ }^{13}$. En la línea de Menéndez Pidal aumenta cada vez más el número de estudiosos, lo que hace que en la actualidad se cuente con interesantes aportaciones en este sentido ${ }^{14}$.

Este punto nos lleva a otro. Admitimos al lado de las nuevas corrientes no una entrada en el vacío, sino una nueva forma de organizar, pero ¿cómo se produjo ésta?.

La terminología albornoziana nos habla de "los míticos pequeños propietarios libres". En primer lugar parten de una entrada en el vacío, en algo deshabitado durante más de siglo y medio. En segundo lugar mantienen una repoblación a través de presuras individuales que generaría esos pequeños propietarios. Así en base a ello, mientras en el resto de Europa se gestaba y se avanzaba hacia la consolidación del Feudalismo, España decía ser un "islote de hombres libres ${ }^{15}$, es decir nos situaban ya de lleno ante la causa que justificaba la no existencia del Feudalismo.

Como venimos diciendo si bien estas teorías han permanecido vigentes durante largo tiempo, hoy sin embargo tienden a periclitar. Un sustancioso número de obras creemos que en los últimos tiempos demuestran sobradamente la existencia de Feudalismo no sólo en Cataluña, sino en el resto de España. Por nuestra parte asentimos en ello, lo que sin más paleativos nos sitúa ya de lleno ante el intento de dar respuesta a la pregunta que primordialmente motiva este análisis ¿Cómo se formó el Feudalismo?.

Planteamos como hipótesis de trabajo primeramente un conocimiento del mismo pero conceptualmente a nivel general, y seguidamente efectuar una traslación a la zona objeto de análisis, es decir Valdeorras.

\footnotetext{
${ }^{13}$ Menéndez Pidal, Repoblación y tradición en la Cuenca del Duero. Enciclopedia Lingüística Hispánica, Vol I 1960.

${ }_{14}$ Vid. por ejemplo S. de Moxó "Repoblación y Sociedad en la España Cristiana Medieval", Madrid 1979, pp. 21-27.

A. Barros García: Estructuras agrarias y de poder en Castilla el ejemplo de Ávila (1085-1320), Salamanca 1983 T. I, pp. III y ss. El citado autor ofrece ejemplos en la zona objeto de estudio que demuestran la clara pervivencia de población a raíz de las campañas de Alfonso I.

C. Estepa Díez: El nacimiento de Castilla y León, siglos VIII-X T. III, pp. 36, Ambito, Valladolid, 1985

${ }^{15}$ C. Sánchez Albornoz: Pequeños propietarios... op. cit.
}

"CUADERNOS DE ESTUDIOS GALLEGOS", Tomo XLI, Fascículo 106, Santiago 1993-94. 
Al menos a nuestro juicio si queremos llegar a un conocimiento íntegro de la formación del Feudalismo, hemos de basarnos en dos obras que se pueden considerar de excepcionales.

La primera de las mismas bajo el título "Formación del Feudalismo en la Península Ibérica", corre a cargo de los Profesores Barbero y Vigil ${ }^{16}$. Exponen y demuestran la formación del Feudalismo a través de la realidad comunitaria. Su teoría refuta por completo esa "repoblación" entendida como una entrada en el vacío y por supuesto esas presuras individuales.

Ven la formación del Feudalismo partiendo de la sociedad esclavista del mundo romano, deteniéndose de un modo particular en el análisis de las sociedades gentilicias del Norte peninsular. Cántabros, vascones y astures, serán pueblos que se mantuvieron independientes durante largo tiempo y diferentes a los demás, por lo tanto con unas estructuras sociales y económicas distintas. A los vascones debe su origen el primitivo reino astur, y por lo tanto éste en su expansión hacia el valle del Duero, lo que transmite será esa base social y económica de las organizaciones gentilicias. Organizaciones que no habían sido eliminadas por la sociedad esclavista que pervivían en el norte peninsular y que paulatinamente se fueron transformando en comunidades de aldea, que en un primer momento mantuvieron su unidad de origen ${ }^{17}$.

Esta unidad de origen acabará rompiéndose al producirse una división de clases dentro de la comunidad, cuyos miembros más ricos serían ahora los únicos depositarios en sus linajes familiares de la antigua unidad de origen y del pasado gentilicio. División en clases que tiene lugar en los siglos altomedievales y la misma lo que provoca es una jerarquización interna en la comunidad. Los señores se van apropiando de los bienes de las mismas por diversos caminos ${ }^{18}$, y todo el proceso genera el triunfo de la gran propiedady por ende el sometimiento de los menos poderosos que quedarán sujetos a relaciones de dependencia. Dicho proceso nos sitúa ya de lleno ante la formación de las estructuras feudales, es decir el fortalecimiento paulatino de los señores. Llegará un momento en que la sociedad

${ }^{16}$ La Formación del Feudalismo en la Peninsula Ibérica, $\mathrm{I}^{\mathrm{a}}$ Ed. 1978, $3^{\mathrm{a}}$ ed. Barcelona 1982

${ }^{17}$ Ibid, pp. 401-402.

${ }^{18}$ Vid. Estepa Díez C., El Nacimiento de León y Catilla siglos VIII-X... op. cit. p. 48. Del mismo autor Formación y desarrollo del Feudalismo Castellano-leonés, I Congreso de Estudios Medievales, León 1987.

"CUADERNOS DE ESTUDIOS GALLEGOS", Tomo XLI, Fascículo 106, Santiago 1993-94. 
estará formada solamente por señores en la cúspide: los auténticos focos de poder feudal, y por los campesinos en la base sometidos a éstos. Cuando esto se produzca netamente estamos ante la consolidación del Feudalismo.

C. Estepa es el autor de la otra obra aludida. Parte del presupuesto de ésta, pero se centra ya en una zona concreta: la castellano-leonesa, e intenta demostrar en la base de la anterior cómo surge y se forma la gran propiedad $^{19}$.

Opera con categorías conceptuales nuevas que él propone, tales como propiedad dominical, dominio señorial y señorío jurisdiccional ${ }^{20}$. Conceptos que responden a la formación de las estructuras feudales, y que cronológicamente pueden corresponderse con distintos momentos según la evolución de dichas estructuras.

De las mismas y por lo que a nosotros respecta dado el período cronológico elegido, nos interesa fundamentalmente la propiedad dominical. Avanzado el tiempo, ya en el siglo XII, más hacia la segunda mitad, se podría aludir a la específica del dominio señorial.

En líneas generales, y en palabras de C. Estepa, la formación del feudalismo pasa por la constitución de la propiedad dominical. La misma la podemos encontrar constatada como tal ya por efecto de esa ruptura de la unidad de origen que provoca la división en clases, o bien seguirla en cuanto a su proceso de formación a través de la disolución de la realidad comunitaria. Cualquiera que sea el camino, la propiedad dominical se gesta a raíz de la disolución comunitaria, disolución que es el eje sobre el que gira la formación del feudalismo.

Propiedad dominical nos dice C. Estepa que viene dada por el poder económico sobre la tierra y los hombres. El propietario de la tierra es el señor que tiene campesinos dependientes a su servicio a los que puede exigir rentas y prestaciones y que no tienen en principio que ver con la propia comunidad de aldea. Serán campesinos dependientes dominicalmente de su señor, distintos a los que engrosan las filas de la comunidad de aldea, en tanto estos eran los campesinos "libres" por supuesto entendidos en el sentido de la comunidad. El paso del tiempo situará a unos y otros en el mismo plano de sumisión y dependencia ${ }^{21}$.

\footnotetext{
${ }^{19}$ Estepa Díez C., En Torno al Feudalismo Hispánico, I Congreso de Estudios Medievales, Fundación Sánchez Albornoz, León 1987.

${ }^{20}$ Vid. la definición de las mismas, citada obra, p. 162.

${ }^{21}$ Ibid, es continuación.
}

"CUADERNOS DE ESTUDIOS GALLEGOS", Tomo XLI, Fascículo 106, Santiago 1993-94. 
A la hora de comprender la formación de la estructura feudal, dos serán los aspectos claves que la determinan: la existencia de propiedad dominical por un lado, y por otro la de comunidades de aldea, fenómenos interrelacionados. El fortalecimiento de los señores, se fue configurando básicamente a través de un proceso de engrosamiento de la propiedad dominical por medio de la disolución de la realidad comunitaria. Se trata pues de seguir cada fenómeno. Quien ostenta dicha propiedad, como se ha ido gestando y en definitiva cual es el alcance de la misma, ello nos situará según sea éste, ante un mayor o menor desarrollo de las estructuras feudales.

Lo expuesto no queda ya sino que trasladarlo a la zona que es objeto de estudio, es decir Valdeorras.

\section{LA FORMACIÓN DEL FEUDALISMO EN VALDEORRAS EN EL PERÍODO ASTUR: SIGLO $X$}

Hemos de advertir que no es mucho lo que podemos adelantar para el conocimiento de la formación del Feudalismo en Valdeorras en este primer momento. Contamos con poquísima documentación exclusivamente del siglo X. Por otro lado se debe de tener en cuenta el desconocimiento general para el período altomedieval desde planteamientos más novedosos. Cada día se sabe más de las comunidades de aldea $^{22}$, pero el letargo y silencio sobre las mismas ha sido muy notorio y esto es algo que no debe de olvidarse.

Partimos del siglo X en Valdeorras y primeramente hemos de pensar en la sociedad que en la misma se está gestando y como ésta va determinando la formación de ese primer momento feudal.

¿Existía propiedad dominical en Valdeorras para el período astur?;

${ }^{22}$ Los profesores B. y Vigil despuntaron en este campo como pioneros con su estudio sobre la Formación... Suscribe lo dicho por ellos Reyna Pastor con su obra sobre "Resistencias y luchas campesinas en la época del crecimiento y consolidación de la formación feudal, Castilla y León, siglos X-XIII, Madrid 1980. Es de destacar el estudio dedicado a las mismas por parte de C. Estepa en su obra citada "el Nacimiento de... y del mismo autor en su obra sobre la formación del Feudalismo... Reyes Tellez dedica un artículo a las comunidades de aldea basado en datos arqueológicos: En el pasado histórico de Castilla y León, I Congreso de Historia de Castilla y León, V. I Burgos 1982.

"CUADERNOS DE ESTUDIOS GALLEGOS", Tomo XLI, Fascículo 106, Santiago 1993-94. 
¿Cómo se gestó en caso de constatarse?. ¿Qué sucedía con la comunidad?... La respuesta a estas y otras preguntas serán la base, en tanto obtengan respuesta, a la formación del Feudalismo.

¿Cómo seguir el proceso de formación de la propiedad dominical?. Son diversos los mecanismos. El siglo XI en la zona nos permitirá una mayor profundización en tanto se dispone de más documentación, pero veamos qué podemos aportar para el período astur.

Transmisiones de carácter regio de bienes junto a los que se incluyen campesinos dependientes nos remiten al campo de la propiedad dominical en algunas zonas próximas a Valdeorras ${ }^{23}$. Para este período no contamos con ninguna referencia de este tipo en Valdeorras.

Otro punto de avance en el sentido de la propiedad dominical parte de la constatación de transmisiones en la misma línea que las anteriores, pero efectuadas por nobles. Zonas próximas nos hablan de ello ${ }^{24}$. Tampoco Valdeorras nos aporta nada en este campo para esta fase.

Otra vía de conocimiento parte de la transmisión de villas. Este es en líneas generales un tema muy delicado y que a veces se nos escapa. Se disponen de ejemplos de transmisiones de villas en las que se notifica la inclusión de campesinos dependientes en las mismas para otras zonas, en la misma línea que los anteriores ${ }^{25}$. Ahora bien no está demás aclarar que esta especificidad en cuanto al campesino dependiente "dominicalmente" en las villas no siempre se da. Son muchas las veces que la documentación nos proporciona transmisiones de villas, pero no se nos dice más sobre las mismas. A sabiendas de lo arriesgado que ello resulta, planteamos como hipótesis, el mismo carácter para éstas que para las anteriores ${ }^{26}$, por su-

${ }^{23}$ Vid. nota 4 de este análisis. Remitimos de todos modos al lector a nuestra Tesis Doctoral, C. III.

${ }^{24}$ Vid. nota 4 citada en la anterior. Igualmente remitimos al lector al citado C. III de nuestra Tesis.

${ }^{25}$ Por ejemplo la citada en la nota 4 que protagoniza el monarca Vermudo II. Ahora bien, como decimos la transmisión de otra serie de villas en las que no se especifican la presencia de campesinos dependientes en las mismas, no es causa justificativa para no pensar en lo contrario. En el año 932 Homar Gudilo y Auria, que son hermanos donan una villa de nombre Cabanilla "cerca de la aldea de S. Justo". B.N. Mss 4357. Tbo Negro ${ }^{\circ}$ 420. En 952 el matrimonio Virtute y Seside donan al Monasterio de S. Cosme y S. Damián "la villa de Arganzuela con su iglesia de S. Pedro". Ibid. n. 365.

${ }^{26}$ Remitimos al contenido de la nota $\mathrm{n}^{\circ} 25$. Algunos ejemplos fuera del Bierzo nos dan pie para la interpretación realizada. Así un documento de 990 hace relación de toda una

"CUADERNOS DE ESTUDios GALLEGOS", Tomo XLI, Fascículo 106, Santiago 1993-94. 
puesto guardando ciertas reservas.

Tampoco para Valdeorras podemos aportar nada en este sentido en el período astur. Avanzado el tiempo, en la fase siguiente esto que se da en otras zonas, se comprobará sucede lo mismo en Valdeorras.

Podemos decir que en el seguimiento de la propiedad dominical como tal constatada y definida, es decir ya formada, no disponemos de dato alguno para Valdeorras. En este sentido estimamos tiene mucho que ver la parquedad de documentación, si bien también se podría pensar en una zona arcaica donde la realidad comunitaria tiene peso específico.

Como ya hemos adelantado un punto es el conocimiento de la propiedad dominical como tal. Los ejemplos expuestos para otras zonas nos hablaban en este sentido. Pero otro punto de conocimiento para la misma es a través de la disolución comunitaria. A medida que esta se produce avanza la gran propiedad, y en este sentido podemos aportar algo para Valdeorras en el período astur.

Disponemos de un documento que data del año 960 y por el que un presbítero "Hanimio" se desprende de un monto considerable de propiedades en la zona a favor de otro monasterio ${ }^{27}$. Es muy importante tener presente la explicación propia de la persona que protagoniza tal acto: un presbitero.

$\mathrm{Al}$ objeto de entender la actuación que ejercen estos presbíteros, muy

serie de villas que son transmitidas y en las que no se especifica la presencia de campesinado dependiente" Aliam villam quam dicunt Sanctum Martinum... Palacio et Cacavolos". El mismo documento a renglón seguido explicita la donación de otras fuera del Bierzo concretamente en Sanabria "de nombre Galende cum suos homines quae ibi solebant exercer servitium". Ello nos puede dar la base para pensar en las anteriores en el mismo sentido. Martínez Martínez M., Cartulario del Monasterio de Santa María de Carracedo, inédito, f. I.

27 "Ecclesia mea propia vocabulo Sancto Vincentio cum suo accessu, vineas, pumares, kasas, cubas, lectos, Kathedras vel omnem meam hereditatem quantum ibidem ad prestandum invenire potueritis. In Casoyo sexta portione ad integram quantum me competet inter meos germanos alia villa qui iacet inter Sancta Cruce et Sancto Vincentio septima portione ab integro in Lamelas mea portione trans monte ad Castro Maiore mea portione de Casoyo usque in rivulo de Guiliellamo mea porcione sexta ab integro. In villa Requisindi duas rationes ad integro de que me competet inter meos heredes ata alia mea apresuta in valle de Arboyro et in illas Penellas agro concluso seminatura ducodecim modios de caraele de Causo usque in monte villa mea ad integrum alium locum ecclesie Sancte Crucis hereditatem quem habeo de meos avos...". I. García Tato, La Documentación Medieval del Tumbo de S. Martín... pp. 23-24.

"CUADERNOS DE ESTUDIOS GALLEGOS", Tomo XLI, Fascículo 106, Santiago 1993-94. 
usual verlos protagonizando este tipo de donaciones no sólo en Valdeorras, sino en prácticamente todas las zonas ${ }^{28}$, hemos de retrotaernos a la fase primera de "colonización". La misma hemos advertido que se entendía en el sentido de los grupos actuando colectivamente. Magnates, Reyes, comunidades monásticas, comunidades campesinas... eran algunos de esos grupos. Las relaciones que mantienen entre ellos en primera instancia nos sitúan ante los primeros brotes de la disolución comunitaria.

Se adelantó la existencia de un abultado número de comunidades monásticas en esta primera fase en una zona próxima a Valdeorras como el Bierzo. Por su parte en Valdeorras parece se constata la existencia tan sólo de tres monasterios como también ya vimos.

Si nos planteamos dar respuesta al origen de los mismos, hemos de partir del presupuesto de que eș variado. Algunos son monasterios propios, otros de carácter fundacional ${ }^{29}$. Sea cual sea cada caso, la situación nos sugiere una pregunta que creemos viene a dar respuesta al cometido que nos estamos proponiendo ¿cabría suponerlos como algo vinculado a la realidad comunitaria?.

En principio en toda esta serie de Monasterios que normalmente se constatan en el período astur no sólo para el Bierzo, Valdeorras, sino de forma general, respetamos el carácter específico propio que cada uno pueda tener. Ahora bien, de cualquier modo que sea, creemos "a priori" que tienen mucho que ver con la realidad comunitaria, en una estrecha relación con las comunidades campesinas que junto a ellos, amén de otros grupos, protagonizaron ese primer momento de "colonización".

Si partimos de este presupuesto, entenderemos el papel que desempeñan toda esa serie de presbíteros de los que reiterativamente nos suele hablar cualquier documentación altomedieval. ¿Qué interpretación darles en el marco de la estructura feudal?.

El paso en el tiempo nos permite ver a miembros dentro de la comuni-

\footnotetext{
${ }^{28}$ Cualquier documentación que se observe de distintos sitios nos habla de ello. Vid. por ejemplo la documentación de la de la Catedral de León de Ruiz Asencio. Tres tomos, León, 1987, o incluso en zonas como Asturias la de la Catedral de Oviedo de García Larragueta, Oviedo 1962.

${ }^{29}$ En este sentido remitimos al lector al C. III citado de nuestra Tesis Doctoral, y más especificamente a un cuadro "Monasterios fundados en el Bierzo desde mediados del siglo $I X$ a mediados del XI, incluído en dicho capítulo. En el mismo se especifica el carácter propio de cada cenobio, así como sus posibles fechas fundacionales.
}

"CUADERNOS DE ESTUDIOS GALLEGOS", Tomo XLI, Fascículo 106, Santiago 1993-94. 
dad en situación privilegiada y que se van disociando de la misma a través de esos actos de donación que encabezan, precisamente de bienes no de escasa significación. Estos miembros destacados son los presbíteros y con su actuación están contribuyendo a la disolución de la comunidad originaria y por ende generando el avance de la gran propiedad que poco a poco va concéntrandose en menos manos. El papel de estos presbíteros ha sido el de intermediarios, en el marco de la comunidad, su importancia estriba en ese momento en ser catalizadores del sistema feudal.

El caso específico de Valdeorras nos habla de un presbítero que dona iglesias, alguna villa, porciones en otras etc. ${ }^{30}$. Bienes como decimos de extrema significación y que nos remiten a este sentido de la comunidad. La fase siguiente — siglo XI—, nos permitirá ahondar con más profusión en esta temática, pues contamos con buena base para llevarlo a cabo.

Al filo de la conclusión para el período astur en Valdeorras advertimos en primer lugar una tónica que tiene mucho que ver con lo que de forma general se conoce para otras zonas. Bien es verdad que en principio la importancia de la zona parece relativa. Nos remite a esta relatividad por ejemplo el escaso número de Monasterios en la misma. Por otro lado no hay que olvidar la escasez de documentación de la que se dispone y que ciertamente no ofrece muchas posibilidades. Por lo demás nos da la sensación de encontrarnos con una zona intermedia, en cierto sentido bastante arcaica y donde la introducción del feudalismo parece lenta. El proceso de avance del mismo tiene mayor peso específico en la realidad comunitaria y su disolución en aras del engrosamiento de la gran propiedad.

\section{VALDEORRAS EN EL SIGLO XI: EL AVANCE EN LA FOR- MACIÓN DE LAS ESTRUCTURAS FEUDALES}

En contraposición al período astur, el siglo XI y primera mitad del XII, se puede considerar en Valdeorras como el período clave en la formación del Feudalismo. Las bases de la etapa precedente, si bien tímidas, inician ahora el camino en el sentido de avance y de lo que posteriormente ha de ser la consolidación del Feudalismo.

${ }^{30}$ Vid. nota 27 donde se explicita exhaustivamente todo el monto de bienes donados por este presbítero.

"CUADERNOS DE ESTUDIOS GALLEGOS", Tomo XLI, Fascículo 106, Santiago 1993-94. 
Donaciones de carácter noble, ya constatadas en esta época, nos sitúan ante un preclaro avance de la propiedad dominical. En la misma línea donaciones de carácter regio, como asimismo donaciones protagonizadas por personas que no denotan una situación privilegiada nos hablan de los restos de la realidad comunitaria.

Nuestro planteamiento de trabajo para esta fase seguirá por las mismas directrices de la anterior. Se intentará demostrar en qué medida se comprueba la existencia de propiedad dominical como tal, o bien como ésta va avanzando a través de la disolución comunitaria.

La tónica que se manifiesta para este período es la ya iniciada en el período astur. Lo más generalizado igual que en ese momento es hablar del avance de la propiedad en el sentido de la disolución comunitaria más que en la consignación ya efectiva de la propiedad.

Una vía que nos situaba ante la propiedad dominical como tal era a través de las transmisiones que ciertos nobles efectuaban de bienes junto a los que incluían campesinos dependientes. Entendida en este sentido, no es el caso para Valdeorras ni en el período astur, ni en la fase subsiguiente. Sin embargo sí que contamos con nobles que protagonizan donaciones de bienes cuya importancia indirectamente nos lleva al campo de la propiedad dominical.

Así las cosas, el siglo XI nos da información en Valdeorras de nobles que donan Monasterios propios como igualmente iglesias. En $1083 \mathrm{D}$. Diego y sus herederos dan sus posesiones en Alijo de Valdeorras. Entre las mismas explicitan "Monasterium nostrum propium quem habemus de abolorum nostrorum... quam vocitant Sancti Martini in territorio Iorres in villa que nuncupant Aliso ${ }^{131}$.

Otros documentos nos hablan de transmisiones de iglesias, lo que indirectamente nos da a entender aunque no se especifique la situación social de la persona que encabeza tal acto, que debido a la naturaleza de lo donado, se encuentra en una situación privilegiada.

En 1092 Tello Eriz y su esposa donan la iglesia y otras posesiones en Jagoaza "concedimus ecclesia nostra propia... vocitant Sancta Maria... territorio valle de Iurres..." ${ }^{132}$. Otra donación de 1096 llevada a cabo por Pelayo Bonelliz y Alvaro Telliz, entre otros bienes objeto de donación p. 37.

${ }^{31}$ Quintana Prieto A., Tumbo Viejo del Monasterio de S. Pedro de Montes, León 1977,

${ }^{32}$ Ibid, p. 142.

"CUAdERNOS DE eSTUdiOS GALlEGOS", Tomo XLI, Fascículo 106, Santiago 1993-94. 
incluye "nostras porciones in ecclesia Sancte Marie... in vale Iurrus ubi dicent Sancte Marie de Heremita" ${ }^{\prime \prime 3}$.

Creemos oportuno ver en estos Monasterios e iglesias focos de concentración de propiedad a los que en principio se les puede suponer la existencia de un campesinado dependiente en su entorno, en tanto emanados de la realidad comunitaria. De ser así, se nos está proyectando una función muy importante en el ejercicio de poder sobre el campesinado por parte de los grupos más privilegiados y en último término un avance de la propiedad dominical en tanto cabe suponerlos como focos de concentración de propiedad con campesinos dependientes.

En el marco de la disolución comunitaria ya en el período astur se habló de la importancia ejercida por toda una serie de presbíteros protagonistas de transmisiones de bienes de cierta significación. Tan sólo un documento nos sirvió de base en el período precedente.

Por el contrario en el momento cronológico que ahora nos ocupa, son diversas las ocasiones en que encontramos a presbíteros en tal situación. En 1028 el presbítero Vela a juzgar por los bienes que dona al Monasterio de San Martín de Castañeda ${ }^{34}$ denota ser evidentemente un gran propietario. En 1075, creemos que se trata del mismo presbítero, dona igualmente un monto considerable de bienes al Monasterio de San Pedro de Montes "villa mea propia cum omnia prestantia quem habemus in territorio Yorres in villam quem vocitant Cassayo..."135.

Millarroso será la localidad en la que otro presbítero, en este caso denominado Constancio, tiene ubicadas un monto considerable de bienes que decide donar al Monasterio de San Pedro de Montes $^{36}$. La prolijidad de documentos en este sentido es para tenerla en cuenta. Aún en el siglo XII se dan donaciones de este tipo ${ }^{37}$.

No se trata de resaltar de nuevo su papel, como se vio de intermedia-

\footnotetext{
${ }^{33}$ Ibid, p. 173.

34 "ipsas hereditates in Casoyo in territorio Sancti Salvatoris et iacent ipsas hereditates in loco predicto super illa carraria andamiosoa que discurret per illo prato a Casoio; corte concluso cum equas, cavallus, aurum... García Tato I., Documentación S. Martín.. p. 25.

${ }^{35}$ Quintana Prieto A., Tumbo Viejo... p. 109.

${ }^{36}$ Ibid, p. 125.

${ }^{37}$ En 1107 el presbítero Costancio da propiedades en Millarroso de Valdeorras y en Quintanilla de Robledo al Monasterio de S. Pedro y Montes. Quintana Prieto A., Tumbo Viejo... p. 214.
}

"CUAdERNOS DE eStudios GAllegOS", Tomo XLI, Fascículo 106, Santiago 1993-94. 
rios. Para este momento nos interesa resaltar esta representatividad de forma reiterativa y que nos está proyectando ante un gran avance de disolución comunitaria.

Otro tipo de donaciones que están protagonizadas por personas que en principio no denotan cierta significación en tanto el bien donado así lo da a entender, nos sitúan en este camino de disolución de la realidad comunitaria. Restos de grupos familiares como "García Moriniz una cum uxor mea Fronille Ananalaz cognomento Sol, et Pelayo Monniuz et Auro Villito et Monniuz... ${ }^{38}$ dan sus bienes al Monaterio de San Pedro de Montes. En 1096 Rodrigo Tellez protagoniza otra donación en este sentido ${ }^{39}$, por citar algunos ejemplos entre otros muchos ${ }^{40}$.

No nos parece muy propicio ver en estas personas "personas libres". Creemos más en la línea de interpretación que ha de venir por el campo de la comunidad en aras de la disolución de la misma. Son por decirlo así, los últimos residuos que van quedando de la comunidad ya a extinguir. Serán los campesinos "libres", pero en el seno de la comunidad.

La via de disolución en este sentido puede ofrecer una mayor variabilidad. Es el caso de la constatación de comunidades enteras que colectivamente y entre las que a veces destaca un miembro en las mismas como más principal, deciden donar un bien común, - normalmente una iglesia - a los que se están imponiendo como focos de poder feudal. Se cuenta con bastantes ejemplos en esta línea para el Bierzo ${ }^{41}$, también para La $\mathrm{Cabrera}^{42}$. Desgraciadamente no disponemos de ninguno para Valdeorras, pero que no dudamos de su existencia, pues es algo generalizado. A través de ese acto colectivo pasan a engrosar las líneas de dependencia quedando por lo tanto desde ese momento insertos y sujetos a los dominios señoriales que se han ido alzando con el poder ${ }^{43}$.

\footnotetext{
${ }^{38}$ Ibid, p. 166.

${ }^{39}$ Ibid, p. 180.

${ }^{40}$ Vid. dcto. n. 91, 92, 93, 77, 84. Quintana Prieto A., Tumbo Viejo...

${ }^{41}$ En 1102 una comunidad de aldea decide donar "su iglesia propia en territorio del Vierzo en la villa de Nozeda al arroyo de Arlanza su titulo de San Pedro con todas sus heredades y pertenenzias. B.N.Mss 4357, Tbo Negro, n. 455. En este sentido vid. el Tumbo de S. Pedro de Montes Documentos n 116, p. 206; dcto. n 161, p. 260; dcto. 256, p. 357.

${ }^{42}$ En 1101 unos vecinos de Corporales dan la iglesia al Monasterio de S. Pedro de Montes. Tumbo Viejo... p. 197.

${ }^{43}$ Reyna Pastor: Resistencias y luchas campesinas...
}

"CUADERNOS DE ESTUDIOS GALLEGOS", Tomo XLI, Fascículo 106, Santiago 1993-94. 
El siglo XI pues nos sitúa para Valdeorras ante una prolongación de lo que había sido la etapa anterior. El proceso de formación feudal avanza en este momento con más fuerza, sin embargo estimamos que la zona está marcada de un cierto arcaismo.

Ya y por lo que respecta al XII, hasta la primera mitad momento en que detenemos este estudio, la tónica que se manifiesta es prácticamente igual que las fases anteriores, si bien en una línea de mayor progreso hacia la consolidación del sistema feudal.

Presbíteros aún encabezan actos de donación ${ }^{44}$. Toda una serie de transacciones nos remiten a esos residuos de grupos familiares en el sentido de la comunidad ${ }^{45}$. Tal vez lo más notable para esta centuria es que se aprecia un cierto protagonismo realengo, tema del que no hemos podido adelantar nada con anterioridad.

No obstante dicho protagonismo parece más acusado a partir de la segunda mitad. Se dispone de documentación a partir de este momento en que se ve a los monarcas efectuando donaciones que en principio nos parecen de suma importancia, pues nos remiten al campo de la propiedad dominical ${ }^{46}$. Otros documentos nos sitúan ante categoría conceptuales más avanzadas en el tiempo como puede ser el dominio señorial ${ }^{47}$. Nuestro propósito es abordar toda esta temática en otro momento, justo a partir a mediados del siglo XII. Lo adelantamos a fin de evitar rupturas cronológicas que pensamos en nada benefician a la investigación.

${ }^{44}$ Remitirse a la nota $n^{0} 37$.

${ }^{45}$ Vid. documentos Tumbo Viejo... $\mathrm{n}^{\circ} 124,125,133,136,145, \ldots$

${ }^{46}$ En 1187 Fernando II dona a Vermudo Fernández S. Esteban de Valdeorras, Petín, Montes y otras localidades "cum suis omnibus directuris et pertinentiis cum Cernego, cum Valleza et cum sua Mata et cum Varzenalla, cum Mones, cum Petin et cum omnibus illis quae ibi as regias pertinent vocem..." Martínez Martínez M., Cartulario Monasterio... fol. 100.

${ }^{47}$ Alfonso VI de la villa de Fervenza de Camba al Monasterio de Santa Maria de Carracedo "cum montibus, fontibus... et omnibus sus suis directuris terminis..." Ibid, fol. 502.

"CUADERNOS DE ESTUdIOS GALLEGOS", Tomo XLI, Fascículo 106, Santiago 1993-94. 